\title{
Severe Symptomatic Hypocalcemia after Denosumab Administration in an End-Stage Renal Disease Patient on Peritoneal Dialysis with Controlled Secondary Hyperparathyroidism
}

\author{
Mohit Agarwal $^{1^{*}}$, Éva Csongrádi ${ }^{1,2}$, Christian A. Koch ${ }^{1}$, Luis A. Juncos ${ }^{1}$, \\ Vonda Echols', Mihály Tapolyai ${ }^{3}$ and Tibor Fülöp ${ }^{1}$. \\ ${ }^{1}$ Department of Medicine, University of Mississippi Medical Center, Jackson, MS, USA. \\ ${ }^{2}$ Department of Medicine, Medical and Health Science Centre University of Debrecen, \\ Hungary. \\ ${ }^{3}$ Fresenius Medical Care, Semmelweis University, Budapest, Hungary.
}

Authors' contributions

This work was carried out in collaboration between all authors. Authors TF and MA designed and wrote most of the case report. Authors MA and VE collected the data. Author MA, TF, $M T$ and ÉC managed the literature searches. Authors CAK, ÉC, MT and LAJ provided intellectual inputs. All authors read and approved the final manuscript.

Case Study

Received $31^{\text {st }}$ December 2012

Accepted $17^{\text {th }}$ March 2013

Published 23 ${ }^{\text {rd }}$ April 2013

\section{ABSTRACT}

We report the $1^{\text {st }}$ case of severe, symptomatic hypocalcemia after denosumab (RANKL inhibitor) treatment in a peritoneal dialysis patient with secondary hyperparathyroidism and osteoporosis. A 58-year-old Caucasian female has been receiving chronic ambulatory peritoneal dialysis for four years secondary to polycystic kidney disease. Laboratory studies revealed: albumin-corrected calcium $9.0 \mathrm{mg} / \mathrm{dL}$, phosphorus $5 \mathrm{mg} / \mathrm{dL}$, alkaline phosphatase (ALP) $58 \mathrm{U} / \mathrm{L}$ [normal, 40-105], albumin $3.4 \mathrm{gm} / \mathrm{dL}$ [normal, 3.6-5.4] and intact parathyroid hormone (PTH) $315 \mathrm{pg} / \mathrm{mL}$ [normal, 40-72]. Marked osteoporosis was noted on the DXA scan, preventing her from renal transplantation considerations. She had failed conventional medical treatment, including per os calcium, monthly ergocalciferol (50,000 units/month), activated vitamin-D analog (doxercalciferol) and 
renal-failure adjusted alendronate (70 $\mathrm{mg}$ twice a month). She was started on subcutaneous denosumab $60 \mathrm{mg}$ every 6 months. After her first dose, she developed a progressive drop of calcium, phosphorus, bicarbonate and magnesium, in spite of massive escalation of doxercalciferol and calcium supplementation. Hypocalcemia nadired at $6.3 \mathrm{mg} / \mathrm{dL}$ with symptomatic tetany, requiring a brief hospitalization approximately 7 weeks after denosumab treatment. Her elevated PTH rose further transiently $(647 \mathrm{pg} / \mathrm{mL})$, along with ALP (123 U/L). Bone-mineral parameters normalized approximately 3 months after denosumab administration. The observed phenomenon resembled the phenotype of "hungry bone syndrome" observed after surgical parathyroidectomy.

Conclusion: Treatment decisions based on bone densitometry results alone are not transposable between patients with or without end-stage renal disease. Denosumab may lead to critical hypocalcemia in dialysis patients and further aggravate existing secondary hyperparathyroidism.

Keywords: Denosumab; end-stage renal disease; hungry bone syndrome; hypocalcemia; osteoporosis; RANK ligand inhibitor; tetany.

\section{INTRODUCTION}

Bisphosphonates bind bone minerals and are absorbed by mature osteoclasts, inducing osteoclast apoptosis and suppressing resorption $[1,2]$. Due to the potential renal toxicity of bisphosphonates, there has been some interest in the use of denosumab, a receptor activator of nuclear factor (NF-KB) ligand (RANKL) inhibitor for treatment of osteoporosis in patients with renal impairment. Until recently, denosumab, as per the manufacturer's instructions, did not require dosage adjustments for renal impairment [3]. Published experience of the use of denosumab in end-stage renal disease (ESRD) is, however, very scant. One preliminary study of 8 hemodialysis patients [4] and another case report have raised concerns about severe hypocalcemia in hemodialysis patients [5]. We report the $1^{\text {st }}$ case of severe symptomatic hypocalcemia secondary to denosumab in a peritoneal dialysisdependent patient with controlled secondary hyperparathyroidism.

\section{CASE STUDY}

Our index patient was a 58-year-old Caucasian female with end-stage renal disease (ESRD) secondary to polycystic kidney, who had been on chronic ambulatory peritoneal dialysis (CAPD) for 4 years and doing well on renal replacement therapy, except for a progressive height loss. A dual-energy x-ray absorptiometry (DXA) scan was obtained in August of 2011, revealing significant osteoporosis (Table. 2). She denied a prior history of osteoporosis or any fracture. Except for continued tobacco abuse, she denied risk factors such as glucocorticoid use, seizure medications or a family history of osteoporosis. She had been on a vitamin D supplement 50,000 International Units once a month and was previously on over-the counter calcium-carbonate as phosphorus binder; however, she developed transient hypercalcemia and calcium carbonate was discontinued. She was placed on alendronate (Fosamax $\left.{ }^{\circledR}\right)$ which she had been taking without any difficulty. 
Table 1. Changes of bone-mineral metabolism parameters after denosumab administration

\begin{tabular}{|c|c|c|c|c|c|c|c|c|c|c|}
\hline $\begin{array}{l}\text { Date (Days } \\
\text { Since } \\
\text { Denosumab } \\
\text { dosage) }\end{array}$ & $\begin{array}{l}04 / 10 / 12 \\
(-73)\end{array}$ & $\begin{array}{l}06 / 21 / 12 \\
(-1)\end{array}$ & $\begin{array}{l}07 / 26 / 12 \\
(34)\end{array}$ & $\begin{array}{l}08 / 14 / 12 \\
(53)\end{array}$ & $\begin{array}{l}08 / 23 / 12 \\
(62)\end{array}$ & $\begin{array}{l}09 / 11 / 12 \\
(81)\end{array}$ & $\begin{array}{l}10 / 09 / 12 \\
(109)\end{array}$ & $\begin{array}{l}11 / 01 / 12 \\
(132)\end{array}$ & $\begin{array}{l}12 / 06 / 12 \\
(167)\end{array}$ & Ref. Range \\
\hline Calcium & 9.2 & 8.5 & 6.9 & 6.6 & 9.6 & 10.0 & 8.9 & 9.7 & 9.3 & $\begin{array}{l}8.2-10.1 \\
\mathrm{mg} / \mathrm{dL}\end{array}$ \\
\hline $\begin{array}{l}\text { Calcium } \\
\text { (Adjusted for } \\
\text { albumin) }\end{array}$ & 9.5 & 9.0 & 7.4 & 7.0 & & 10.6 & 9.5 & 10.3 & 9.8 & $\begin{array}{l}8.2-10.1 \\
\mathrm{mg} / \mathrm{dL}\end{array}$ \\
\hline Phosphorus & 5.0 & 5.0 & 3.4 & 4.6 & 2.6 & 4.2 & 5.7 & 7.5 & 7.6 & $2.5-5.0 \mathrm{mg} / \mathrm{dL}$ \\
\hline $\begin{array}{l}\text { Alkaline } \\
\text { Phosphatase }\end{array}$ & 58 & & 85 & 123 & & 122 & 62 & 50 & 43 & 40-105 IU/L \\
\hline iPTH & 315 & & 647 & & & & 117 & & & $14-72 \mathrm{pg} / \mathrm{mL}$ \\
\hline
\end{tabular}

Table 2. DXA results summary (August 2011)

\begin{tabular}{|c|c|c|c|c|c|}
\hline Region & Area $\left(\mathrm{cm}^{2}\right)$ & BMC (g) & BMD $\left(\mathrm{g} / \mathrm{cm}^{2}\right)$ & T-score & Z-score \\
\hline Neck & 5.27 & 2.53 & 0.480 & -3.3 & -2.2 \\
\hline Total & 32.05 & 20.68 & 0.645 & -2.4 & -1.7 \\
\hline L1 & 13.38 & 8.79 & 0.657 & -3.0 & -2.0 \\
\hline L2 & 14.24 & 9.48 & 0.666 & -3.3 & -2.1 \\
\hline L3 & 16.94 & 12.42 & 0.734 & -3.2 & -2.0 \\
\hline L4 & 16.09 & 10.52 & 0.654 & -3.7 & -2.4 \\
\hline Total & 60.65 & 41.22 & 0.680 & -3.3 & -2.1 \\
\hline
\end{tabular}


However, she noted further height loss over the ensuing months and was also rejected by the renal transplant team, citing her severe osteoporosis ruling out considerations of renal transplant eligibility. Co-morbid issues associated with her ESRD included secondary hyperparathyroidism and hyperphosphatemia, renal anemia with functional iron deficiency and chronic hypoalbuminemia and hypokalemia associated with peritoneal dialysis. Other co-morbidities included a prior cerebrovascular accident with no residual deficit, wellcontrolled hypertension, allergic rhinitis, chronic anxiety, history of colonic polyps and arteriovenous malformations and vitamin-D deficiency requiring replacement. For chronic metabolic acidosis, she was taking potassium-citrate on a long-term basis.

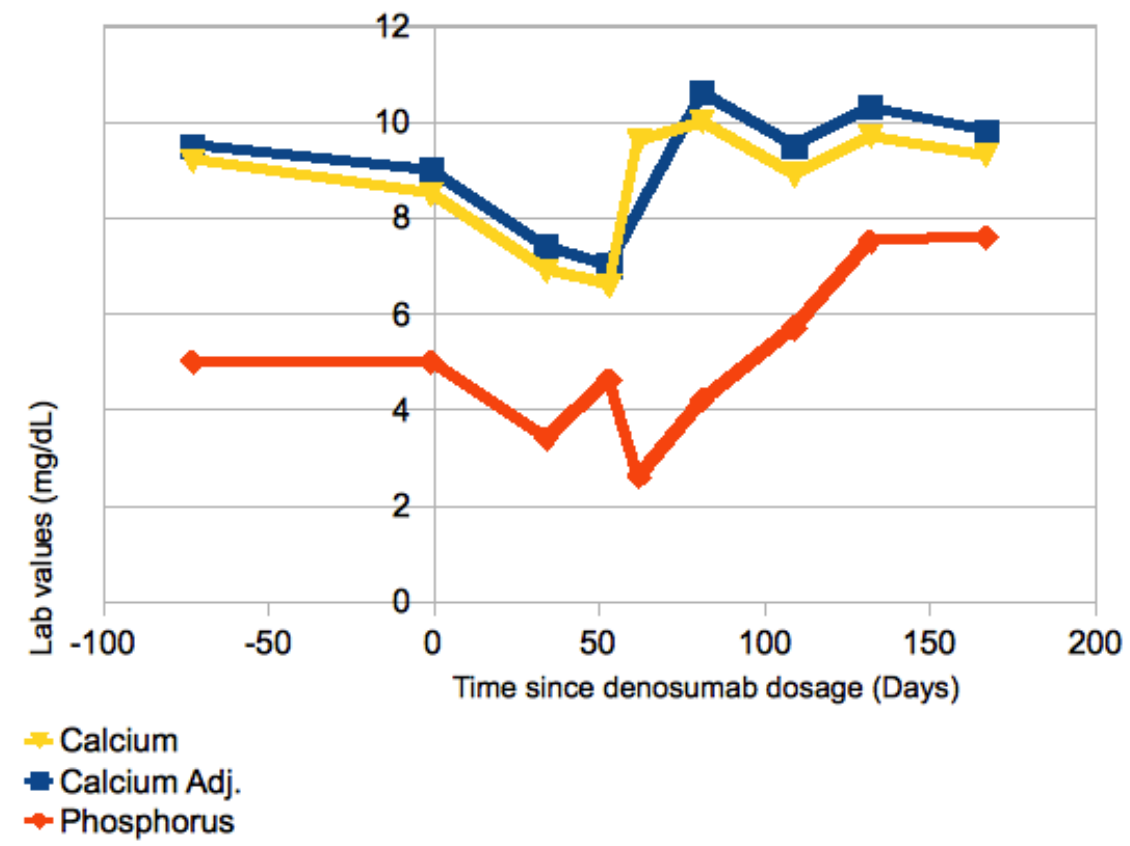

Fig. 1. Calcium, adjusted calcium and phosphorus changes after denosumab administration.

Denosumab initiated on day '0', alendronate discontinued on day '-31' (31 days prior to denosumab initiation)

In terms of ESRD, she had done reasonably well on a CAPD regimen of 2 liters $x 4$ exchanges with $1.5 \%$ dextrose solution. Return volumes were always satisfactory, resulting in a net ultrafiltration of $300-800 \mathrm{~mL}$ per exchange. Comprehensive dialysis flow sheets revealed excellent Kt/V's between 2.2-2.8 per week but a progressive loss of creatinine clearance over the years, most recently $52 \mathrm{~L} /$ week $/ 1.73 \mathrm{~m}^{2}$. Her residual urine output was very limited (30-40 $\mathrm{mL} / \mathrm{day})$ and contributed little to her overall clearance.

Laboratory studies (Table. 1) at baseline revealed: albumin-corrected calcium $9.0 \mathrm{mg} / \mathrm{dL}$, phosphorus $5 \mathrm{mg} / \mathrm{dL}$, alkaline phosphatase (ALP) $58 \mathrm{U} / \mathrm{L}$ [normal, 40-105], albumin 3.4 $\mathrm{gm} / \mathrm{dL}$ [normal, 3.6-5.4] and intact parathyroid hormone (PTH) $315 \mathrm{pg} / \mathrm{mL}$ [normal, 40-72]. The 25-hydroxy vitamin-D level was normal at $31.3 \mathrm{ng} / \mathrm{ml}$ [normal, 20-100], 1,25 di-hydroxy Vitamin D was $25 \mathrm{pg} / \mathrm{mL}$ [normal, 18-78]. Four years earlier, serum protein and urine electrophoresis revealed only non-selective proteinuria and no evidence of multiple 
myeloma. Baseline medications included doxercalciferol ( $2.5 \mathrm{mcg}$ twice weekly), sevelamer (2400 mg TID with meals and $1600 \mathrm{mg}$ at bedtime), ergocalciferol (50,000 IU once a month) and alendronate (70 $\mathrm{mg}$ twice a month). Rheumatology consultants recommended to discontinue her alendronate and to start denosumab every 6 months. The first dose was given on 06/22/12 along with instructions to take daily over-the-counter $500 \mathrm{mg}$ calcium carbonate. After her first dose, she developed a progressive drop of calcium (Fig. 1), phosphorus, bicarbonate and magnesium, in spite of massive escalation of bioactive vitamin-D analog and calcium supplementation (calcium acetate $2001 \mathrm{mg}$ (elemental calcium $507 \mathrm{mg}$ ) three times daily, calcium carbonate $2250 \mathrm{mg}$ at bedtime (elemental calcium $898.2 \mathrm{mg}$ ), doxercalciferol $2.5 \mathrm{mcg}$ daily) and increased calcium content of peritoneal fluid to $3.0 \mathrm{mEq} / \mathrm{L}$. With progressive hypocalcemia, calcium dropped to $6.3 \mathrm{mg} / \mathrm{dL}$, with symptomatic tetany requiring a brief hospitalization locally approximately 7 weeks after denosumab treatment. As expected, PTH rose transiently during this period $(647 \mathrm{pg} / \mathrm{mL})$, along with ALP (123 U/L). After a short period of transient hypercalcemia, bone-mineral parameters, including calcium, phosphorus and ALP normalized approximately 3 months after denosumab administration. Incidentally, she had an episode of back pain after sudden jerking movements of her extremities and torso in July 2012 leading to an x-ray investigation $(8 / 14 / 12)$ showing right superior and inferior pubic rami fractures which demonstrated healing on follow-up X-rays.

\section{DISCUSSION}

Denosumab is the first Federal Drug Administration (FDA) approved RANK Ligand inhibitor. As Prolia ${ }^{\circledR}$ (Thousand Oaks, CA, USA), it was initially approved in June 2010 for treatment of postmenopausal osteoporosis with high risk of fractures. This approval was based on a three-year, randomized, double-blind, placebo-controlled trial of 7,808 osteoporotic postmenopausal women ages 60 to 91 years (Fracture Reduction Evaluation of Denosumab in Osteoporosis Every 6 Months or FREEDOM Study), in which denosumab reduced the incidence of vertebral, non-vertebral, and hip fractures [6]. Indications since then have been expanded to include increasing bone mass in patients at high risk for fracture receiving androgen deprivation therapy for non-metastatic prostate cancer or adjuvant aromatase inhibitor therapy for breast cancer (Approval September 2011), and to increase bone mass in men with osteoporosis at high risk for fracture (Approval September 2012). As Xgeva ${ }^{\circledR}$, Denosumab is also approved for prevention of skeletal-related events in patients with bone metastases from solid tumors with the exception of Multiple Myeloma (Approval November 2010)

Osteoclast differentiation factor, also called receptor activator of nuclear factor (NF-kB) ligand (RANKL), stimulates the differentiation of osteoclast progenitors into osteoclasts. Osteoprotegerin acts as a natural soluble decoy receptor to bind RANKL and thus, reducing RANKL binding to RANK and reducing bone resorption. Denosumab is a full length human monoclonal antibody of IgG2 subtype mimicking the action of natural osteoprotegerin by binding to and inhibiting RANKL. It exhibits a nonlinear, dose dependent pharmacokinetics after subcutaneous injection, with levels peaking in 7-21 days [7]. As expected for an IgG antibody, it is not cleared by the kidneys and, therefore, does not require dosage adjustments for renal impairment [4]. Denosumab administration results in rapid and sustained decrease for markers of bone resorption; in one study, an $85 \%$ reduction in serum C-telopeptide of type I collagen (a bone resorption marker reflecting osteoclast activity) was seen in 3 days, with a peak reduction in one month [3]. Therefore, this potent and long lasting effect on bone remodeling raises the concerns for hypocalcemia. This was clinically noted in the FREEDOM trial in which within one month, $1.7 \%$ of subjects from the drug arm 
experienced calcium values below $8.5 \mathrm{mg} / \mathrm{dl}$ as opposed to $0.4 \%$ in the placebo arm $[6,8]$. It should be noted that in the FREEDOM Trial, only 73 women had a calculated creatinine clearance 15 to $29 \mathrm{~mL} / \mathrm{min}$ and none had end-stage renal dysfunction $(<15 \mathrm{~mL} / \mathrm{min})$ and, therefore, it was underpowered to study the hypocalcemic effects of denosumab in severe CKD or terminal renal failure [9]. In patients with normal kidney function, nadir in serum calcium is noted on day 10 [3]. This hypocalcemic effect appears to be more pronounced in patients with renal insufficiency. In the FREEDOM Extension study of 4,550 subjects, a more marked reduction in serum calcium was noted in subjects with renal insufficiency: $-5.5 \%$ reduction in mean serum calcium in subjects with creatinine clearance $<30 \mathrm{~mL} / \mathrm{min}$ vs. $3.1 \%$ in subjects with creatinine clearance $\geq 30 \mathrm{~mL} / \mathrm{min}$ at approximately day $10[3,10]$. In another study of 55 patients with various degrees of renal impairment, severe and persistent hypocalcemia was noted in subjects with severe CKD (GFR $<30 \mathrm{~mL} / \mathrm{min} / 1.73 \mathrm{~m}^{2}$ ), which was especially profound with kidney failure requiring hemodialysis. 5 out of 8 dialysis dependent patient developed hypocalcemia (serum calcium $<8 \mathrm{mg} / \mathrm{dL}$ ) and calcium dropped below $7.5 \mathrm{mg} / \mathrm{dL}$ in 2 of them [4]. Additionally, McCormick et al. also recently reported a case of severe hypocalcemia with denosumab in a hemodialysis patient [5]. Interestingly, a recent case report mentioned successful treatment of immobilization-related hypercalcemia with denosumab in a patient with advanced renal insufficiency [11]. In a boy with fibrous dysplasia, denosumab did not impair healing of a femoral fracture that occurred while on treatment during which, similar to our case presented here, secondary hyperparathyroidism developed [12].

Clinical experience thus far suggests that denosumab can result in a rapid and sustained reduction in serum calcium in the presence of severe renal insufficiency or renal failure. This effect may be more pronounced in patients with a history of secondary hyperparathyroidism, perhaps as a function of a larger osteoblast mass in these patients. Inhibition of osteoclastic activity by denosumab may result in excess unopposed osteoblastic activity and resultant severe sustained hypocalcemia, analogous to what we observe as "hungry bone syndrome" after surgical parathyroidectomy [13,14]. This sustained hypocalcemia can also (further) elevate PTH and aggravate existing secondary hyperparathyroidism. Of note, our patient with existing secondary hyperparathyroidism had previously been treated with a bisphosphonate (alendronate) before denosumab had been administered while 1,25dihydroxy vitamin $\mathrm{D}$ and $25-\mathrm{OH}$ vitamin $\mathrm{D}$ levels were within normal range, possibly suggesting an additive effect of both antiresorptive agents. Our patient had PTH in the target range, normal calcium and vitamin $D$ levels at baseline, and was already receiving calcium supplementation and activated vitamin D analog (doxercalciferol) for secondary hyperparathyroidism. While hypocalcemia was expected, the magnitude and the length of the process were certainly surprising to us. Of note, Health Canada placed a hypocalcemia alert on denosumab in June of 2012 (http://www.hc-sc.gc.ca/dhp-mps/medeff/advisoriesavis/public/ 2012/xgeva pc-cp-eng.php). Additionally, the manufacturer (Amgen) also issued additional warning in the US in September of 2012 (http://www.proliahcp.com/pdf/dear healthcare professional letter.pdf), including indicating the risk of severe hypocalcemia.

Our patient was treated for presumed osteoporosis with denosumab per recommendation by the Rheumatologist on the basis of an abnormal DXA scan. As opposed to non-CKD patients in whom osteoporosis is the predominant cause of bone disease and fractures, renal osteodystophy is a heterogenous disorder. Standard imaging diagnostic criteria for osteopenia/osteoporosis developed for use with the general population are sometimes extrapolated to patients with advanced CKD with less than satisfactory results. Bone mineral density (BMD) obtained from the widely used test DXA scan is a prime example. A low bone 
density noticed on DXA is routinely labeled as osteopenia/osteoporosis based upon Word Health Organization (WHO) definitions, but it may be too simple of a label for a patient with advanced CKD $[15,16]$. DXA scan is a two-dimensional projection that indirectly represents BMD through X-ray absorption and as three-dimensional structures are invisible, this imaging modality cannot distinguish between a fully mineralized porous bone and an undermineralized bone with less porosity. Further, DXA scans have not been validated to be a reliable test in dialysis related, or secondary hyperparathyroidism-related bone disease [17]. Disease spectrum in these patients can vary from a high turnover disease with true secondary hyperparathyroidism to a low turnover states with adynamic bone disease and the correct diagnosis in the absence of a bone biopsy remains a challenge. Adynamic bone diseases is of particular concern as the standard antiresorptive treatment prescribed for osteoporosis treatment (bisphosphonates, denosumab) has theoretical potential to exacerbate adynamic bone disease. Even an increase in measured bone density may not result in reduction in fracture risk if the bone is of poor quality. Because of this complex interplay, not all CKD patients are likely to benefit from the standard antiresorptive treatment prescribed for the presumed diagnosis of osteopenia/osteoporosis based upon WHO definition and indeed studies have demonstrated this heterogeneity $[18,19]$.

Currently, we do not have any current guidelines about e.g. adjusting the initial dose of denosumab during the first treatments. Denosumab should be best avoided, for the time being, in patients with ESRD and significant secondary hyperparathyroidism until further clinical experience is been gathered on how to prevent or manage hypocalcemia. Even when patients are adequately repleted on vitamin-D (normal 25-hydroxy vitamin-D and 1,25 di-hydroxy vitamin $D$ levels), large doses of calcium supplements are required. We would like to advocate the use of IV calcium gluconate, supraphysiologic dialysate calcium concentration, and high doses of oral calcium supplements (6-12 grams per day) together with Vitamin $\mathrm{D}$ repletion and reducing denosumab dose under these circumstances. Nonetheless, a blank elimination of this medication from the therapeutic armamentarium may not be the best long-term policy after all; if so doing, we would never expose patients to surgical parathyroidectomy for the fear of hypocalcemia and hungry bone syndrome. The very fact that our patient developed such severe degree of hypocalcemia may serve as a predictor of an excellent potential for bone mineralization and successful future outcomes for bone integrity. We also sorely need bone histology data in these patients, where local expertise on obtaining and interpreting bone biopsy specimens are available. Whether denosumab has any role in treating hypercalcemia in dialysis patients, beyond cancerinduced hypercalcemia [20], is unclear at this time. Potentially, a patient with osteoporosis and hypercalcemia may be the ideal candidate, but this issue is clearly not well studied or understood at this point.

\section{CONCLUSION}

Treatment decisions based on bone densitometry results alone (rather than bone biopsy) are not transposable between patients with or without end-stage renal disease. An antiresorptive agent such as denosumab may lead to critical and prolonged hypocalcemia in dialysis patients as observed in this case and further aggravate existing secondary hyperparathyroidism. Additional research is needed to identify the highest risk patients and effective strategies to minimize this serious side effect. 


\section{CONSENT}

Written informed consent was obtained from the patient for publication of this case report, who had an opportunity to read and review this paper and received a copy of it.

\section{ETHICAL APPROVAL}

This case report is not an animal experiment or experimental study. The University of Mississippi's Institutional Review Board reviewed this project and determined that would not qualify as research (IRB File \#2013-0016). Informed consent was obtained from the patient and we have a copy of the patient's written consent on our files, available for inspection upon request.

\section{ACKNOWLEDGEMENT}

We wish to thank llena S. Agarwal, who has worked closely with the first author and who critically read the manuscript in every draft and helped prepare it for publication.

\section{COMPETING INTERESTS}

Authors have declared that no competing interests exist.

\section{REFERENCES}

1. Hanley DA, Adachi JD, Bell A, Brown V. Denosumab: mechanism of action and clinical outcomes. Int J Clin Pract; 2012.

2. Anastasilakis AD, Polyzos SA, Anastasilakis CD, Toulis KA, Makras P. Denosumab and bisphosphonates: Rivals or potential "partners"? A "hybrid" molecule hypothesis. Med Hypotheses. 2011;77(1):109-11.

3. Prolia (denosumab), prescribing information. Thousand Oaks, Calif.: Amgen; September 2012 (revised).

4. Block GA, Bone HG, Fang L, Lee E, Padhi D. A single-dose study of denosumab in patients with various degrees of renal impairment. J Bone Miner Research. 2012;27(7):1471-9.

5. McCormick BB, Davis J, Burns KD. Severe hypocalcemia following denosumab injection in a hemodialysis patient. Am J Kidney Dis; 2012.

6. Cummings SR, Martin JS, McClung MR, Siris ES, Eastell R, Reid IR, et al. Denosumab for prevention of fractures in postmenopausal women with osteoporosis. N Eng J Med. 2009;361(8):756-65.

7. Body JJ, Facon T, Coleman RE, Lipton A, Geurs F, Fan M, et al. A study of the biological receptor activator of nuclear factor-kappaB ligand inhibitor, denosumab, in patients with multiple myeloma or bone metastases from breast cancer. Clin Cancer Res. 2006;12(4):1221-8.

8. Anastasilakis AD, Toulis KA, Polyzos SA, Anastasilakis CD, Makras P. Long-term treatment of osteoporosis: safety and efficacy appraisal of denosumab. Ther Clin Risk Management. 2012;8:295.

9. Jamal SA, Ljunggren Ö, Stehman-Breen C, Cummings SR, McClung MR, Goemaere $S$, et al. Effects of denosumab on fracture and bone mineral density by level of kidney function. J Bone Miner Res. 2011;26(8):1829-35. 
10. Papapoulos S, Chapurlat R, Libanati C, Brandi ML, Brown JP, Czerwiński E, et al. Five years of denosumab exposure in women with postmenopausal osteoporosis: Results from the first two years of the FREEDOM extension. J Bone Miner Res. 2012;27(3):694-701 .

11. de Beus E, Boer WH. Denosumab for treatment of immobilization-related hypercalcaemia in a patient with advanced renal failure. Clin Kidney J. 2012;5(6):56671.

12. Boyce AM, Chong WH, Yao J, Gafni RI, Kelly MH, Chamberlain CE, et al. Denosumab treatment for fibrous dysplasia. J Bone Mineral Res. 2012;27(7):1462-70.

13. Pierroz DD, Bonnet N, Baldock PA, Ominsky MS, Stolina M, Kostenuik PJ, et al. Are osteoclasts needed for the bone anabolic response to parathyroid hormone? A study of intermittent parathyroid hormone with denosumab or alendronate in knock-in mice expressing humanized RANKL. J Biol Chem. 2010;285(36):28164-73.

14. Witteveen J, van Thiel S, Romijn JA, Hamdy N. Hungry bone syndrome: still a challenge in the post-operative management of primary hyperparathyroidism. A systemic review of the literature. Eur J Endocrinol; 2012.

15. Miller PD. Treatment of osteoporosis in chronic kidney disease and end-stage renal disease. Curr Osteoporos Rep. 2005;3(1):5-12.

16. Moe S, Drüeke T, Cunningham J, Goodman W, Martin K, Olgaard K, et al. Definition, evaluation, and classification of renal osteodystrophy: a position statement from Kidney Disease: Improving Global Outcomes (KDIGO). Kidney Int. 2006;69(11):194553.

17. Jamal SA. Bone mass measurements in men and women with chronic kidney disease. Curr Opin Nephrol Hypertens. 2010;19(4):343-8.

18. Cunningham J, Sprague SM, Cannata-Andia J, Coco M, Cohen-Solal M, Fitzpatrick L, et al. Osteoporosis in chronic kidney disease. Am J Kidney Dis. 2004;43(3):566.

19. Ott S. Review article: Bone density in patients with chronic kidney disease stages 4-5. Nephrology. 2009;14(4):395-403.

20. Santarpia L, Koch CA, Sarlis NJ. Hypercalcemia in cancer patients: pathobiology and management. Horm Metab Res. 2010;42(3):153-64.

(C) 2013 Agarwal et al.; This is an Open Access article distributed under the terms of the Creative Commons Attribution License (http://creativecommons.org/licenses/by/3.0), which permits unrestricted use, distribution, and reproduction in any medium, provided the original work is properly cited.

Peer-review history:

The peer review history for this paper can be accessed here: http://www.sciencedomain.org/review-history.php?iid=205\&id=12\&aid=1275 